\title{
Skeletal muscle PI3K p110ß regulates expression of AMP-activated protein kinase
}

\author{
Ronald W. Matheny Jr. ${ }^{a, \#}$, Mary N. Abdalla ${ }^{\text {a }}$, Alyssa V. Geddis ${ }^{\text {a }}$, Luis A. Leandry ${ }^{\text {a }}$, and \\ Christine M. Lynch ${ }^{\text {a }}$
}

Running title: PI3K p110ß regulates AMPK expression

${ }^{a}$ Military Performance Division, US Army Research Institute of Environmental
Medicine, 10 General Greene Ave, Building 42, Natick, MA, 01760 USA.

\# Correspondence to: Ronald W. Matheny Jr., Military Performance Division, US Army Research Institute of Environmental Medicine, 10 General Greene Ave, Building 42, Natick, MA, 01760, USA, Tel.: (508) 233-5420; Fax: (508) 233-4195; email: ronald.w.matheny.civ@ mail.mil 


\section{Abstract}

Skeletal muscle metabolic homeostasis is maintained through numerous biochemical and physiological processes. Two principal molecular regulators of skeletal muscle metabolism include AMP-activated protein kinase (AMPK) and phosphatidylinositol 3-kinase (PI3K); however, PI3K exists as multiple isoforms, and specific metabolic actions of each isoform have not yet been fully elucidated in skeletal muscle. Given this lack of knowledge, we performed a series of experiments to define the extent to which PI3K p110 $\beta$ mediated expression and (or) activation of AMPK in skeletal muscle. To determine the effect of p110 $\beta$ inhibition on AMPK expression and phosphorylation in cultured cells, C2C12 myoblasts were treated with a pharmacological inhibitor of p110 $($ TGX-221), siRNA against p110 3 , or overexpression of kinase-dead p110ß. Expression and phosphorylation of AMPK were unaffected in myoblasts treated with TGX-221 or expressing kinase-dead p110 $\beta$. However, expressions of total and phosphorylated AMPK at T172 were reduced in myoblasts treated with p110 $\beta$ siRNA. When normalized to expression of total AMPK, phosphorylation of AMPK S485/491 was elevated in p110 $\beta$-deficient myoblasts. Similar results were observed in tibialis anterior muscle from mice with conditional deletion of $\mathrm{p} 110 \beta$ (p110 $\beta-\mathrm{mKO}$ mice). Analysis of AMPK transcript expression revealed decreased expression of Prkaa2 in p110 $\beta$-deficient myoblasts and in p110 3 -mKO muscle. Loss of p110 $\beta$ had no effect on oligomycin-stimulated phosphorylation of AMPK or phosphorylated Acetyl-CoA carboxylase (ACC), although oligomycin-induced AMPK and ACC phosphorylation were increased in p110 $\beta$-deficient myoblasts compared to oligomycinstimulated control myoblasts when normalized to levels of total AMPK or ACC. Overall, these results suggest that $\mathrm{p} 110 \beta$ positively regulates expression of AMPK in cultured myoblasts and in skeletal muscle in vivo; moreover, despite the reduced abundance of AMPK in p110 $\beta$-deficient 
myoblasts, loss of p110 $\beta$ does not appear to impair AMPK activation following stimulus. These findings thus reveal a novel role for $\mathrm{p} 110 \beta$ in mediating skeletal muscle metabolic signaling.

Key Words: AMP-activated Protein Kinase (AMPK); phosphoinositide 3-kinase (PI3K); myoblast; skeletal muscle 


\section{Introduction}

Metabolic homeostasis in skeletal muscle is maintained through the balanced integration of numerous biochemical, genetic, and cell-signaling processes. One well-studied molecular mediator of skeletal muscle energy metabolism is AMP-activated protein kinase (AMPK), a heterotrimeric serine/threonine kinase that regulates cellular energy homeostasis by modulating metabolic and energetic processes [1]. AMPK is activated under conditions of low cellular energy (high AMP:ATP ratio) such as seen with strenuous physical activity $[2,3,4]$. The AMPK complex itself is composed of $\alpha, \beta$, and $\gamma$ subunits, each encoded by two or more genes, thus allowing for a number of heterotrimeric combinations [5]. Among these subunits, the $\alpha$ subunit contains the kinase domain, the $\beta$ subunit contains the glycogen binding domain, and the $\gamma$ subunit contains adenine nucleotide binding domains. Activation of AMPK is controlled in part by various upstream kinases that phosphorylate specific residues on the $\alpha$ subunit; namely, threonine 172 (T172) and serine 485 in AMPK $\alpha 1$ and serine 491 in AMPK $\alpha 2$ (S485/491) [6]. Phosphorylation at $\mathrm{T} 172$ is generally associated with activation of AMPK, whereas phosphorylation at S485/491 is generally associated with AMPK inhibition [7,8,9]. While a number of molecules that regulate AMPK expression and activation have been identified, much still remains unknown.

Other molecules that regulate skeletal muscle metabolism have been identified, including phosphoinositide 3-kinase (PI3K). Class IA PI3Ks are heterodimeric enzymes composed of a $110 \mathrm{kDa}$ catalytic subunit (p110) and a $85 \mathrm{kDa}$ regulatory subunit (p85) that regulate multiple cellular processes, including metabolism [10]. Three Class IA PI3K p110 catalytic isoforms have been identified in skeletal muscle ( $\alpha, \beta$, and $\delta$ ) [11], and while PI3K p110 $\alpha$ is the principal p110 isoform regulating signaling through receptor tyrosine kinases (RTKs) such as the insulin 
and IGF-I receptors [12,13], p110 is the principal p110 isoform regulating G-protein coupled receptor (GPCR) signaling [14]. Following ligand binding to receptors, PI3K is recruited to activated RTKs or GPCRs via regulatory subunit binding to adaptor proteins. Once in proximity to inositol lipids within the cell membrane, the p110 catalytic subunit phosphorylates the D3 position of the inositol ring, thus generating phosphatidylinositol-(3,4,5)-trisphosphate $\left(\mathrm{PIP}_{3}\right)$ from phosphatidylinositol-(4,5)-bisphosphate $\left(\mathrm{PIP}_{2}\right)$. Generation of $\mathrm{PIP}_{3}$ then allows for recruitment and activation of signaling molecules such as Akt, whose activation regulates growth, differentiation, and metabolism [15]. In general, the role of PI3K in regulating skeletal muscle metabolism is beginning to be elucidated; however, the roles of the specific PI3K p110 catalytic subunit isoforms in regulating muscle metabolism are not well defined.

While both PI3K and AMPK are known regulators of skeletal muscle metabolism, there is currently little information linking specific PI3K p110 catalytic isoforms to AMPK expression or activation. While a recent report has shown that PI3K p110 $\alpha$ negatively regulates phosphorylation of AMPK at T172 and positively regulates phosphorylation of AMPK at S485/491 in C2C12 myoblasts [16], it is unknown whether the other Class IA PI3K isoforms, such as p110ß, regulate skeletal muscle AMPK in a similar fashion. We therefore performed a series of experiments designed to determine whether $\mathrm{p} 110 \beta$ regulates AMPK expression and phosphorylation in skeletal muscle.

\section{Materials and Methods}

Materials and Reagents. C2C12 cells and DMSO were purchased from American Type Culture Collection (ATCC, Manassas, VA). Dulbecco's modified Eagle's mediun (DMEM), fetal 
bovine serum (FBS), penicillin/streptomycin, trypsin, Lipofectamine 2000, and Opti-mem medium were purchased from Life Technologies (Carlsbad, CA). TGX-221 was from Selleck Chemicals (Houston, TX). Antibodies for p-AMPK Thr 172 (\#2535), p-AMPK Ser 485/491 (\#4185), total AMPKa (\#2603), p-Akt Ser 473 (\#4060), total (pan) Akt (\#4685), p110ß (\#3011), GAPDH (\#2118), $\alpha$-tubulin (11H10) (\#2125) and oligomycin (\#9996) were purchased from Cell Signaling Technologies (Danvers, MA).

Cell culture and pharmacological inhibitors. C2C12 were grown in DMEM containing $10 \%$ FBS and $1 \%$ antibiotics at $37^{\circ} \mathrm{C}$ in $5 \% \mathrm{CO}_{2}$. All experiments were performed within six passages of receipt from the vendor (ATCC). TGX-221was dissolved in DMSO and diluted in culture medium such that cells were exposed to a final DMSO concentration of $0.02 \%$. Control cells received an equivalent concentration of DMSO.

Small-interfering RNA (siRNA). Non-targeting control siRNA (catalog number 4390483) and siRNA directed against Pik3cb (p110ß; catalog number s93108) were purchased from Life Technologies. siRNA transfections were carried out as previously described [17].

Baculoviruses and transductions. Generation and transduction of baculoviral constructs were performed exactly as described previously [18].

Animals and tissue harvest. Mice with conditional deletion of Pik3cb in skeletal muscle ('p110ß-mKO') have been described previously [18]. Tibialis anterior muscles were removed from 21 month old $\mathrm{p} 110 \beta$-mKO and floxed littermate control mice, immediately snap-frozen in liquid nitrogen, and stored at $-80^{\circ} \mathrm{C}$ until use. All experimental procedures utilizing mice were conducted under the guidelines on humane use and care of laboratory animals for research and in 
accordance with the Animal Care and Use Committee of the US Army Research Institute of Environmental Medicine.

Protein extraction and immunoblotting. Extraction of protein from cells was performed as described [17], and protein concentrations were determined by the method of Bradford [19]. Western immunoblotting was performed exactly as described previously [18], and densiometric analysis was performed using NIH Image J 1.60 (http://rsbweb.nih.gov/ij/).

RNA isolation, cDNA synthesis, and real-time PCR. RNA isolation from cells and tissues and cDNA synthesis was performed as described previously $[18,20]$ using Trizol reagent (Life Technologies). Real time PCR was performed as previously described [18] normalizing to Hprt1 gene expression. Primers/probes for real-time PCR were purchased from Life Technologies: Prkaal (Mm01296700_m1), Prkaa2 (Mm01264789_m1), and Hprt1 (Mm01545399_m1).

Statistics. Data are presented as mean \pm S.E.M. Statistics were performed using Student's t-test or one-way ANOVA with Tukey's test performed post hoc. A P-value<0.05 was considered significant.

\section{Results}

Loss of PI3K p110ß is associated with altered AMPK protein expression and phosphorylation

Previous research has shown that in cultured myoblasts, PI3K p110 $\alpha$ negatively regulates AMPK phosphorylation at T172 and positively regulates AMPK phosphorylation at S485/491 [16]. To determine the degree to which $\mathrm{p} 110 \beta$ also regulates phosphorylation of AMPK in 
myoblasts, we first treated C2C12 myoblasts with TGX-221, a pharmacological inhibitor of p110 $\beta$ catalytic activity [21,22]. We found that phosphorylation of AMPK at T172, phosphorylation of AMPK at S485/491, and expression of total AMPK were all unaffected by 4hours treatment with $1 \mu \mathrm{M}$ TGX-221 (Figure 1A). Treatment with TGX-221 for 24-hours yielded similar results to those observed following 4-hours treatment (data not shown). Next, C2C12 myoblasts were transduced with cDNA constructs encoding either wild-type p110 $\beta$

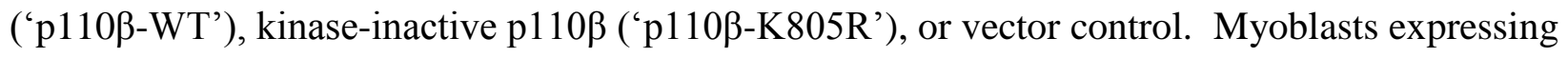

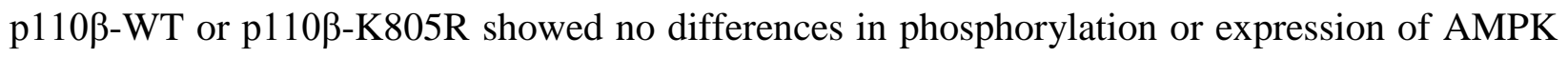
compared to myoblasts transduced with vector control (Figure 1B). Finally, myoblasts were treated with siRNA directed against p110ß. Compared to myoblasts treated with control siRNA, p110ß-deficient myoblasts possessed 52\% less phosphorylated AMPK T172 $(\mathrm{P}<0.05)$ and 43\% less total AMPK $(\mathrm{P}<0.001)$ when normalized to GAPDH expression (Figure 1C and 1D). Phosphorylation at $\mathrm{S} 485 / 491$ was increased $40 \%$ p110ß-deficient myoblasts as compared to control myoblasts when expressed relative to GAPDH $(\mathrm{P}<0.05)$. When phosphorylated AMPK T172 and S485/491 were normalized to total AMPK, there was no difference in T172 phosphorylation between control and p110 $\beta$-deficient myoblasts; however, phosphorylation at S485/491 was increased $\sim 2.5$-fold in cells deficient in p110 $\beta$ compared to control cells (Figure 1E).

Phosphorylation of AMPK at S485/491 has been shown to be mediated by Akt $[8,16]$, and p110ß-deficient myoblasts have previously been shown to possess increased levels of phosphorylated Akt compared to control myoblasts [23]. As figures $1 \mathrm{C}$ and $1 \mathrm{~F}$ show, there was a $76 \%$ increase in Akt phosphorylation at S473 in p110ß-deficient myoblasts compared to control myoblasts $(\mathrm{P}<0.01)$, suggesting that the increased AMPK S485/491 phosphorylation is 
secondary to increased Akt activation. All together, these data reveal that inhibition of p110 $\beta$ catalytic activity alone (through pharmacological inhibition or overexpression of kinase inactive p1 $10 \beta$ ) is not sufficient to alter phosphorylation or expression of AMPK. Instead, it is the loss of p110 $\beta$ protein (through siRNA directed against p110 $\beta$ ) that is associated with these phenomena.

To assess whether these cell-based observations also occurred in vivo, we analyzed levels of phosphorylated and total AMPK in tibialis anterior (TA) muscles of mice with conditional

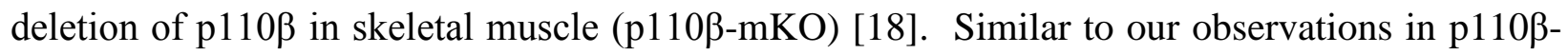
deficient $\mathrm{C} 2 \mathrm{C} 12$ cells, total and phosphorylated AMPK at T172 were each reduced by $37 \%$ in muscles of p110 $\beta$-mKO mice compared to floxed controls when normalized to $\alpha$-tubulin $(\mathrm{P}<0.05$ for both; Figure $1 \mathrm{G}$ and $1 \mathrm{H})$. There was no significant difference in phosphorylation of AMPK

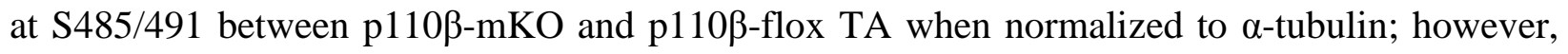
when normalized to total AMPK, TA muscles from p110 $\beta$-mKO mice showed a 2.4-fold increased AMPK S485/491 expression compared to TA muscles from p110 $\beta$-flox mice $(\mathrm{P}<0.01$; Figure 1I). These data reveal that, similar to cultured p110 $\beta$-deficient myoblasts, AMPK phosphorylation and expression are modified in skeletal muscle lacking p110 $\beta$ in vivo.

\section{mRNA expression of Prkaa2 is decreased in p110ß-deficient myoblasts and p110ß-mKO muscle}

We next asked whether the reduced AMPK protein expression observed in p110 $\beta$ deficient myoblasts and in p110 $\beta$-null mouse skeletal muscle was associated with reduced expression of AMPK RNA transcripts. The $\alpha$-subunit of AMPK is encoded by two genes: AMPKa1 (Prkaal) and AMPKa2 (Prkaa2). We found that expression of Prkaa2, but not 
Prkaal, was reduced by $19 \%$ in p110ß-deficient myoblasts as compared to control myoblasts $(\mathrm{P}<0.001$; Figure 2A). Moreover, expression of Prkaa2 was reduced by $9 \%$ in TA muscle of p110ß-mKO mice as compared to $\mathrm{p} 110 \beta$-flox control mice ( $\mathrm{P}<0.05$; Figure $2 \mathrm{~B})$.

\section{Activation of AMPK is not impaired in p110ß-deficient myoblasts.}

Given the altered phosphorylation and reduced abundance of AMPK in unstimulated p110 $\beta$-deficient myoblasts and in p110 $\beta$-mKO mouse skeletal muscle, we next asked whether stimulation of AMPK was affected by $\mathrm{p} 110 \beta$ loss. To test this, we treated control or p110ßdeficient myoblasts with oligomycin, a known activator of AMPK. Oligomycin stimulated AMPK T172 phosphorylation to similar degrees in both control myoblasts and in p110ßdeficient myoblasts when normalized to GAPDH expression ( $\sim 1.6$-fold and $\sim 1.8$-fold for control and p110ß-deficient myoblasts, respectively) (Figure 3A and 3B). Additionally, oligomycininduced phosphorylation of AMPK S485/491 was increased 1.6-fold in control myoblasts $(\mathrm{P}<0.05)$ and $\sim 2.2$-fold in $\mathrm{p} 110 \beta$-deficient myoblasts $(\mathrm{P}<0.001)$ when normalized to GAPDH (Figure 3A and 3B). Levels of total AMPK did not differ between control cells treated with oligomycin and control cells treated with solvent; however, in p110ß-deficient myoblasts, oligomycin treatment further reduced expression of total AMPK beyond that seen with p110 $\beta$

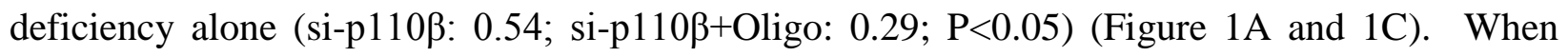
normalized to expression of total AMPK, levels of phosphorylated AMPK at T172 and S485/491 were increased $\sim 6$-fold and $\sim 7.9$-fold, respectively, in oligomycin-stimulated p110 $\beta$-deficient myoblasts as compared to control myoblasts $(\mathrm{P}<0.001$ for each; Figure 3C). 
To determine whether activation of AMPK was altered in response to oligomycin, we examined the expression and phosphorylation of acetyl-CoA carboxylase (ACC), a direct substrate of AMPK. We found that oligomycin treatment increased phosphorylation of ACC at S79 by $~ 2.7$-fold in both control myoblasts and in p110 $\beta$-deficient myoblasts when normalized to expression of GAPDH ( $\mathrm{P}<0.05$ for each) (Figure 3A and 3D). Interestingly, we also observed a $35 \%$ and a $45 \%$ decrease in expression of total ACC in unstimulated and in oligomycin-treated p110 $\beta$-deficient myoblasts, respectively, when normalized to GAPDH expression $(\mathrm{P}<0.05$ for each). When normalized to expression of total ACC, levels of phosphorylated ACC at S79 were increased 5.4-fold in oligomycin-stimulated p110 $\beta$-deficient myoblasts as compared to control myoblasts $(\mathrm{P}<0.01$; Figure 3D). These data demonstrate that oligomycin-stimulated phosphorylation and activation of AMPK is not impaired in p110 $\beta$-deficient myoblasts, even though these cells possess significantly less total AMPK than control myoblasts.

\section{Discussion}

The role of $\mathrm{PI} 3 \mathrm{~K}$ in regulating skeletal muscle metabolism is only beginning to be defined. In this study, we found that PI3K p110 $\beta$ regulated expression of phosphorylated and total AMPK in cultured myoblasts and in mouse skeletal muscle. Of significant interest were our findings that AMPK phosphorylation was not modified in $\mathrm{C} 2 \mathrm{C} 12$ myoblasts treated with TGX221 or expressing kinase-dead (K805R) p110ß, but that AMPK phosphorylation was modified in myoblasts treated with siRNA against p110 $\beta$ (Figure 1). These distinct findings may be explained by the methods used to inhibit p110ß; in particular, the manner in which these methods affect the abundance of the $\mathrm{p} 110 \beta$ protein itself. Indeed, while pharmacological 
inhibition of $\mathrm{p} 110 \beta$ inhibits $\mathrm{p} 110 \beta$ catalytic activity, it does not affect total $\mathrm{p} 110 \beta$ protein levels. Likewise, over-expression of kinase-dead $\mathrm{p} 110 \beta$ results in a greater number of $\mathrm{p} 110 \beta$ molecules overall due to the expression of endogenous $\mathrm{p} 110 \beta$ in addition to the ectopically-expressed p110 $\beta$. However, targeting $110 \beta$ by means of siRNA reduces not only p110 $\beta$ molecules, but also the kinase activity associated with the molecules subjected to the knockdown. Thus, of these three experimental manipulations (chemical inhibition, ectopic expression, and siRNA), only siRNA reduces target protein abundance below endogenous levels. Given the properties of these three experimental manipulations, and given that only siRNA treatment was associated with altered AMPK phosphorylation and expression, our observations suggest that $\mathrm{p} 110 \beta$ may regulate AMPK through a mechanism independent of its kinase activity in myoblasts.

It has been previously shown that loss of $\mathrm{p} 110 \beta$ protein results in compensatory upregulation of $\mathrm{p} 110 \alpha$ and Akt activities in $\mathrm{C} 2 \mathrm{C} 12$ myoblasts [23]. It is this $\mathrm{p} 110 \alpha$ hyperactivation that likely underlies the decreased phosphorylation of AMPK T172 and increased phosphorylation of AMPK S485/491 we observed in p110 3 -deficient myoblasts (Figure 1). Indeed, it has been reported that phosphorylation of AMPK T172 was reduced and phosphorylation of AMPK S485/491 was increased in C2C12 myoblasts expressing a constitutively-active mutant of $\mathrm{p} 110 \alpha[16]$. Together, these data suggest that the altered phosphorylation of AMPK in p110 $\beta$-deficient myoblasts is modulated principally through $\mathrm{p} 110 \alpha$ hyper-activation resulting from the reduced expression of $\mathrm{p} 110 \beta$. Despite this similarity between p110ß-deficient myoblasts and myoblasts expressing constitutively-active p110 $\alpha$ in modulating AMPK phosphorylation, we found that total AMPK protein was reduced in p110ß-deficient myoblasts - an effect not observed in myoblasts expressing constitutively-active p110 [16].

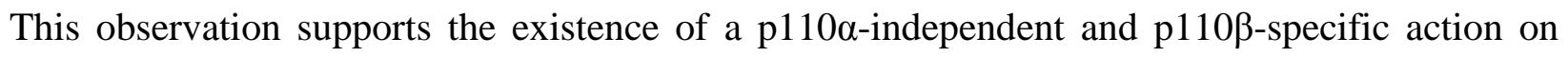


AMPK. The reduction in AMPK protein appears to be dependent on the abundance of $\mathrm{p} 110 \beta$ protein and not $\mathrm{p} 110 \beta$ catalytic activity, as AMPK protein levels were unaffected by pharmacological inhibition or over-expression of p110 $\beta$ cDNA constructs.

To further study the mechanism underlying the reduced total AMPK protein expression in p110ß-deficient myoblasts and mouse skeletal muscle, we asked whether the reduced total AMPK protein expression might result from reduced transcript expression of AMPK $\alpha$ RNA. To this end, we examined expression of Prkaal and Prkaa2 in p110ß-deficient myoblasts and in muscles of mice with conditional deletion of $\mathrm{p} 110 \beta$ in skeletal muscle. Our findings revealed that the mRNA abundances of Prkaa2 were significantly reduced in p110ß-deficient C2C12 cells and in muscles of p110 $\beta$-mKO mice (Figure 2). However, this reduced expression was quite modest (19\% in p110 - -deficient cells and $9 \%$ in $\mathrm{p} 110 \beta \mathrm{mKO}$ muscle), and may not fully explain the $\sim 50 \%$ reduced expression of AMPK protein. This discrepancy between the small reductions in mRNA and larger reductions in AMPK protein may result from post-transcriptional events (such as a reduction in the rate of nuclear export of AMPK transcripts), or from translational or posttranslational events affecting the rate of protein synthesis, stability, or turnover. In any case, previous evidence suggests that mRNA levels may explain only $40 \%$ of the variability in protein levels globally [24]; thus, while our data clearly show that $\mathrm{p} 110 \beta$ regulates expression of AMPK, further research must be undertaken to establish the mechanism(s) underlying this phenomenon.

In response to oligomycin treatment, phosphorylation of AMPK at T172 and at S485/491 were increased to similar degrees in p110 $\beta$-deficient myoblasts as they were in control myoblasts, even though p110 $\beta$-deficient myoblasts possessed less total AMPK protein than did control myoblasts (Figure 3). This suggests that loss of p110 $\beta$ has no effect on oligomycin- 
stimulated AMPK phosphorylation. However, a second and equally valid interpretation of these findings is that oligomycin-induced phosphorylation of AMPK is actually increased compared to control myoblasts treated with oligomycin because these phosphorylated AMPK molecules represent a greater proportion of total AMPK molecules in p110 $\beta$-deficient myoblasts than they do in control myoblasts (Figure 3C). It could therefore be argued that phosphorylation of AMPK is more sensitive to oligomycin treatment in p110 $\beta$-deficient myoblasts than in control myoblasts. Such an increased response to oligomycin in p110ß-deficient myoblasts could be caused by stimulation of an AMPK kinase or inhibition of an AMPK phosphatase, although further study is needed to determine the exact mechanism regulating the increased oligomycininduced AMPK phosphorylation (as a function of total AMPK) in myoblasts lacking p110 $\beta$.

In conclusion, our data suggest that $\mathrm{p} 110 \beta$ positively regulates expression of AMPK in cultured myoblasts and in mouse skeletal muscle, possibly through a mechanism independent of its catalytic activity. Despite the reduced abundance of AMPK in p110ß-deficient myoblasts, phosphorylation of AMPK was sensitive to oligomycin to the same degree as control myoblasts, indicating that p110 $\beta$ deficiency did not impair AMPK activation in myoblasts. In all, we have identified a novel role for PI3K p110 $\beta$ in regulating AMPK expression and phosphorylation in skeletal muscle, thus identifying $\mathrm{p} 110 \beta$ as a contributor to metabolic signaling. This information may ultimately be used to inform treatment strategies to optimize skeletal muscle health during disease states. 


\section{Acknowledgements}

M.N.A., A.V.G., and C.M.L. were supported by appointments to the Postgraduate Research Participation Program at the U.S. Army Research Institute of Environmental Medicine administered by the Oak Ridge Institute for Science and Education through interagency agreement between the U.S. Department of Energy and U.S. Army Medical Research and Materiel Command. The opinions or assertions contained herein are the private views of the authors and are not to be construed as official or reflecting the views of the Army or the Department of Defense. Any citations of commercial organizations and trade names in this report do not constitute an official Department of the Army endorsement of approval of the products or services of these organizations. 


\section{References}

[1] K.A. Coughlan, R.J. Valentine, N.B. Ruderman, A.K. Saha, AMPK activation: a therapeutic target for type 2 diabetes?, Diabetes Metab Syndr Obes 7 (2014) 241-253.

[2] Z.P. Chen, T.J. Stephens, S. Murthy, B.J. Canny, M. Hargreaves, L.A. Witters, B.E. Kemp, G.K. McConell, Effect of exercise intensity on skeletal muscle AMPK signaling in humans, Diabetes 52 (2003) 2205-2212.

[3] W.W. Winder, D.G. Hardie, Inactivation of acetyl-CoA carboxylase and activation of AMPactivated protein kinase in muscle during exercise, Am J Physiol 270 (1996) E299-304.

[4] J.F. Wojtaszewski, P. Nielsen, B.F. Hansen, E.A. Richter, B. Kiens, Isoform-specific and exercise intensity-dependent activation of 5'-AMP-activated protein kinase in human skeletal muscle, J Physiol 528 Pt 1 (2000) 221-226.

[5] M.C. Towler, D.G. Hardie, AMP-activated protein kinase in metabolic control and insulin signaling, Circ Res 100 (2007) 328-341.

[6] D.G. Hardie, The AMP-activated protein kinase pathway--new players upstream and downstream, J Cell Sci 117 (2004) 5479-5487.

[7] S. Horman, D. Vertommen, R. Heath, D. Neumann, V. Mouton, A. Woods, U. Schlattner, T. Wallimann, D. Carling, L. Hue, M.H. Rider, Insulin antagonizes ischemia-induced Thr172 phosphorylation of AMP-activated protein kinase alpha-subunits in heart via hierarchical phosphorylation of Ser485/491, J Biol Chem 281 (2006) 5335-5340.

[8] J. Ning, G. Xi, D.R. Clemmons, Suppression of AMPK activation via S485 phosphorylation by IGF-I during hyperglycemia is mediated by AKT activation in vascular smooth muscle cells, Endocrinology 152 (2011) 3143-3154. 
[9] R.J. Valentine, K.A. Coughlan, N.B. Ruderman, A.K. Saha, Insulin inhibits AMPK activity and phosphorylates AMPK Ser(4)(8)(5)/(4)(9)(1) through Akt in hepatocytes, myotubes and incubated rat skeletal muscle, Arch Biochem Biophys 562 (2014) 62-69.

[10] E. Hirsch, C. Costa, E. Ciraolo, Phosphoinositide 3-kinases as a common platform for multihormone signaling, J Endocrinol 194 (2007) 243-256.

[11] B. Geering, P.R. Cutillas, G. Nock, S.I. Gharbi, B. Vanhaesebroeck, Class IA phosphoinositide 3-kinases are obligate p85-p110 heterodimers, Proc Natl Acad Sci U S A 104 (2007) 7809-7814.

[12] L.C. Foukas, P.R. Shepherd, Phosphoinositide 3-kinase: the protein kinase that time forgot, Biochem Soc Trans 32 (2004) 330-331.

[13] Z.A. Knight, B. Gonzalez, M.E. Feldman, E.R. Zunder, D.D. Goldenberg, O. Williams, R. Loewith, D. Stokoe, A. Balla, B. Toth, T. Balla, W.A. Weiss, R.L. Williams, K.M. Shokat, A pharmacological map of the PI3-K family defines a role for p110alpha in insulin signaling, Cell 125 (2006) 733-747.

[14] C. Murga, S. Fukuhara, J.S. Gutkind, A novel role for phosphatidylinositol 3-kinase beta in signaling from G protein-coupled receptors to Akt, J Biol Chem 275 (2000) 1206912073.

[15] B.D. Manning, L.C. Cantley, AKT/PKB signaling: navigating downstream, Cell 129 (2007) 1261-1274.

[16] R.W. Matheny, Jr., A.V. Geddis, M.N. Abdalla, L.A. Leandry, Phosphatidylinositol 3kinase p110alpha mediates phosphorylation of AMP-activated protein kinase in myoblasts, Biochem Biophys Res Commun 469 (2016) 1117-1122. 
[17] R.W. Matheny, Jr., M.L. Adamo, Effects of PI3K catalytic subunit and Akt isoform deficiency on mTOR and p70S6K activation in myoblasts, Biochem Biophys Res Commun 390 (2009) 252-257.

[18] R.W. Matheny, Jr., M.A. Riddle-Kottke, L.A. Leandry, C.M. Lynch, M.N. Abdalla, A.V. Geddis, D.R. Piper, J.J. Zhao, Role of phosphoinositide 3-OH kinase p110beta in skeletal myogenesis, Mol Cell Biol 35 (2015) 1182-1196.

[19] M.M. Bradford, A rapid and sensitive method for the quantitation of microgram quantities of protein utilizing the principle of protein-dye binding, Anal Biochem 72 (1976) 248254.

[20] R.W. Matheny, Jr., M.L. Adamo, PI3K p110 alpha and p110 beta have differential effects on Akt activation and protection against oxidative stress-induced apoptosis in myoblasts, Cell Death Differ 17 (2010) 677-688.

[21] S.P. Jackson, S.M. Schoenwaelder, I. Goncalves, W.S. Nesbitt, C.L. Yap, C.E. Wright, V. Kenche, K.E. Anderson, S.M. Dopheide, Y. Yuan, S.A. Sturgeon, H. Prabaharan, P.E. Thompson, G.D. Smith, P.R. Shepherd, N. Daniele, S. Kulkarni, B. Abbott, D. Saylik, C. Jones, L. Lu, S. Giuliano, S.C. Hughan, J.A. Angus, A.D. Robertson, H.H. Salem, PI 3kinase p110beta: a new target for antithrombotic therapy, Nat Med 11 (2005) 507-514.

[22] C.M. Lynch, L.A. Leandry, R.W. Matheny, Jr., Lysophosphatidic acid-stimulated phosphorylation of PKD2 is mediated by PI3K p110beta and PKCdelta in myoblasts, J Recept Signal Transduct Res 33 (2013) 41-48.

[23] R.W. Matheny, Jr., C.M. Lynch, L.A. Leandry, Enhanced Akt phosphorylation and myogenic differentiation in PI3K p110beta-deficient myoblasts is mediated by PI3K p110alpha and mTORC2, Growth Factors 30 (2012) 367-384. 
[24] B. Schwanhausser, D. Busse, N. Li, G. Dittmar, J. Schuchhardt, J. Wolf, W. Chen, M. Selbach, Global quantification of mammalian gene expression control, Nature 473 (2011) $337-342$. 


\section{Figure Legends}

Figure 1. Reduced expression of AMPK in p110 $\beta$-deficient myoblasts and in mouse skeletal muscle lacking p110ß. (A) C2C12 myoblasts were treated with DMSO or $1 \mu \mathrm{M}$ TGX221 for 4-hours. Western blotting was performed on lysates using the indicated antibodies. (B) Myoblasts were transduced with vector control ('Vector'), wild-type p110 $\beta$ ('p110 $\beta$-WT'), or kinase-inactive p110 ('p110 $\beta$-K805R') as described in the Methods section. Lysates were immunoblotted with the indicated antibodies. Blots are representative of four independent experiments. (C) Myoblasts were treated with $5 \mathrm{nM}$ non-targeting control siRNA ('si-Con') or 5 nM siRNA directed against p110 ('si-p110 $\beta$ ') as described in Methods. Lysates were immunoblotted with the indicated antibodies. Blots are representative of three independent experiments. (D) Quantification of phosphorylated and total AMPK normalized to GAPDH (shown in panel C) (means \pm SEMs; $\mathrm{n}=3$; asterisks indicate significant difference from si-Con myoblasts; *, $\mathrm{P}<0.05$; ***, $\mathrm{P}<0.001)$. (E) Quantification of phosphorylated AMPK normalized to expression of total AMPK (all shown in panel C) (means \pm SEMs; $n=3$; asterisks indicate significant difference from si-Con myoblasts; *, P<0.05). (F) Quantification of phosphorylated Akt normalized to expression of total Akt shown in panel $\mathrm{C}$ (means $\pm \mathrm{SEM} ; \mathrm{n}=3$; asterisks indicate significant difference from si-Con myoblasts; **, $\mathrm{P}<0.01$ ). $\quad$ (G) Western immunoblotting was performed on lysates from TA muscle from p110 $\beta$-flox and p110 $\beta$-mKO mice using the indicated antibodies. (H) Quantification of phosphorylated and total AMPK normalized to $\alpha$ tubulin (all shown in panel G) (means \pm SEMs; $n=9$ p110 $\beta$-flox and 11 p110 $\beta$ mKO; asterisks indicate significant difference from p110 -flox; *, P<0.05). (I) Quantification of phosphorylated AMPK normalized to total AMPK (all shown in panel G) (means \pm SEMs; $\mathrm{n}=9$ 
p110 $\beta$-flox and 11 p110 $\beta$-mKO; asterisks indicate significant difference from p110 $\beta$-flox; **, $\mathrm{P}<0.01)$.

Figure 2. Expression of Prkaal and Prkaa2 in p110ß-deficient myoblasts and in TA muscle of p110 $\beta-m K O$ mice. (A) Real-time PCR was performed on cDNA derived from control or p110 $\beta$-deficient myoblasts using the indicated primers/probes (means \pm SEMs; $n=3$; asterisks indicate significant difference from si-Con-treated myoblasts; ***, $\mathrm{P}<0.001$ ). (B) Real-time PCR was performed on cDNA derived from TA muscle of p110 $\beta$-flox or p110 $\beta$-mKO mice using the indicated primers/probes (means \pm SEMs; $n=6$ p110 $\beta$-flox and 7 p110 3 -mKO; asterisks indicate significant difference from p110 $\beta$-flox; *, P<0.05).

Figure 3. Effects of oligomycin on AMPK activation in p110ß-deficient myoblasts. (A) Control or p110 $\beta$-deficient myoblasts were treated with $0.5 \mu \mathrm{M}$ oligomycin for 30-min, harvested for protein lysates, and Western blotting performed using the indicated antibodies. Blots are representative of three independent experiments. (B) Quantification of phosphorylated and total AMPK normalized to GAPDH from panel A (means \pm SEMs; $n=3$; one-way ANOVA with Tukey test; asterisks indicate significant difference from non-targeting siRNA-treated control ('si-Con') myoblasts *, $\mathrm{P}<0.05$; **, $\mathrm{P}<0.01$; ***, $\mathrm{P}<0.001$ ). (C) Quantification of phosphorylated AMPK normalized to expression of total AMPK from panel A (means \pm SEMs; $\mathrm{n}=3$; one-way ANOVA with Tukey test; asterisks indicate significant difference from control (si-Con) myoblasts ***, $\mathrm{P}<0.001)$. (D) Quantification of phosphorylated ACC normalized to expression of GAPDH and to total ACC from panel A (means \pm SEMs; $n=3$; one-way ANOVA with Tukey test; asterisks indicate significant difference from control (si-Con) myoblasts; *, $\mathrm{P}<0.05 ; * *, \mathrm{P}<0.01)$. 
A

B

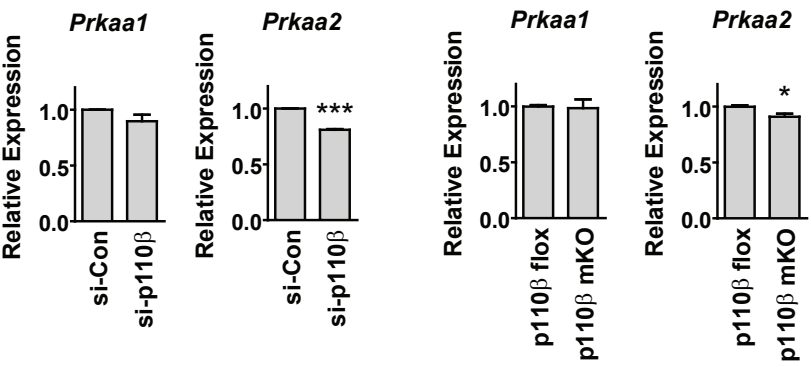


A
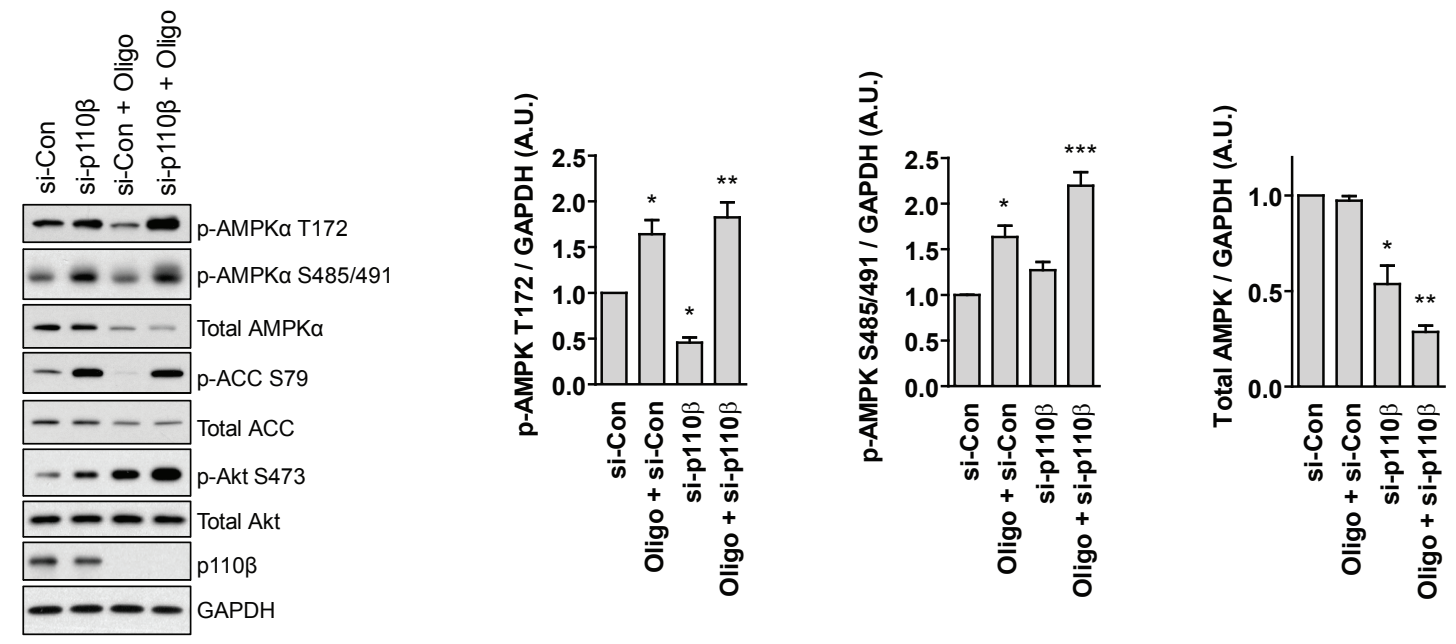

C

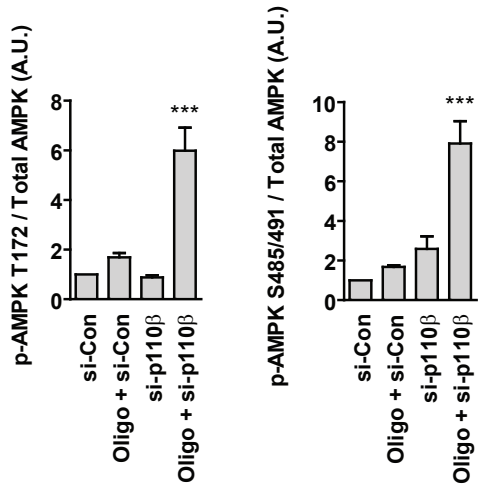

D

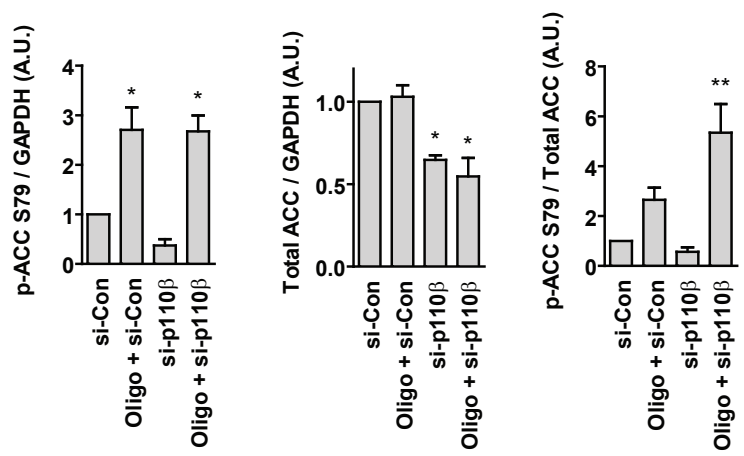

